\title{
Catalytic and thermal hydrocarbonation of methyleneaziridines
}

\author{
Byoung Ho Oh, Itaru Nakamura, and Yoshinori Yamamoto* \\ Department of Chemistry, Graduate School of Science, Tohoku University, Sendai 980-8578 \\ Japan \\ E-mail: yoshi@yamamoto1.chem.tohoku.ac.jp
}

Dedicated to Professor Keiichiro Fukumoto on the occasion of his $70^{\text {th }}$ birthday

(received 19 May 03; accepted 30 June 03; published on the web 04 July 03)

\begin{abstract}
Reaction of the methyleneaziridine 1 with carbon pronucleophiles (2, $\left.\mathrm{H}-\mathrm{CR}_{3}\right)$ proceeds smoothly in the presence of a palladium catalyst affording the corresponding hydrocarbonation products 5 in good to high yields. In the absence of palladium catalysts, the reaction of $\mathbf{1 a}$ with $2 \mathbf{a}$ at $120^{\circ} \mathrm{C}$ afforded the ring opened product $\mathbf{9}$ in good yield.
\end{abstract}

Keywords: Methyleneaziridine, pronucleophile, palladium, hydrocarbonation

\section{Introduction}

2-Methyleneaziridines are small-ring compounds containing a nitrogen atom, which have high ring strain. It is known that the ring opening of methyleneaziridines with Grignard reagents (or organolithium compounds), ${ }^{1 \mathrm{a}-\mathrm{d}}$ acid chlorides, ${ }^{\text {e-f }}$ and $\mathrm{HCl}^{1 \mathrm{~g}}$ occurs through $\mathrm{N}-\mathrm{C} 3$ bond cleavage (eq 1), while ring opening with $\mathrm{HOPh}$ proceeds through $\mathrm{N}-\mathrm{C} 2$ bond cleavage ${ }^{\text {1h }}$ (eq 2). Accordingly, the normal reaction of 2-methyleneaziridines with nucleophiles produces ringopened derivatives. Recently we reported that the reaction of the methyleneaziridines $\mathbf{1}$ with carbon pronucleophiles 2 proceeds smoothly in the presence of a palladium catalyst to give, in good- to high yields, the ring products 5 (eq 3). ${ }^{2}$ Formally, this is a hydrocarbonation reaction of the double bond of $\mathbf{1}$ with carbon pronucleophiles. In this paper, we report the detailed study of the palladium-catalyzed hydrocarbonation of methyleneaziridines together with an attempt at asymmetric hydrocarbonation using chiral phosphine ligands. 

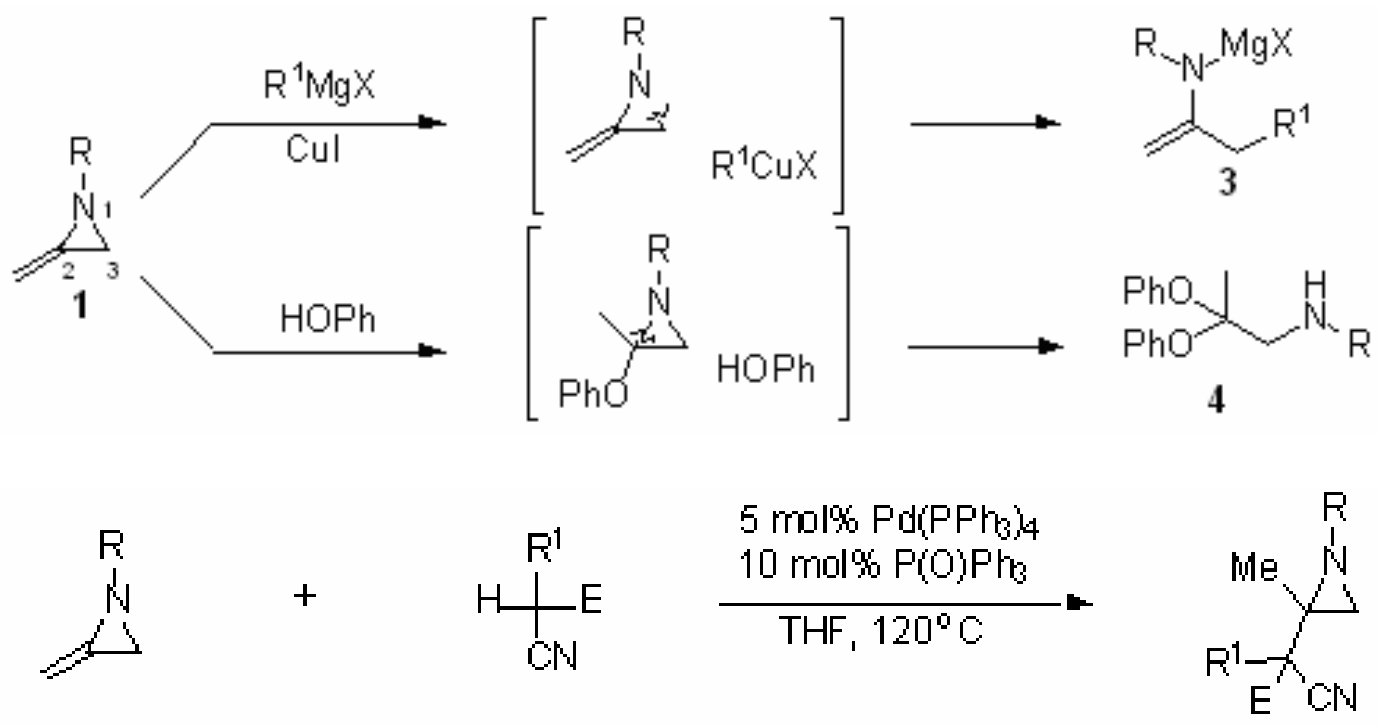
1a; $\mathrm{R}=\mathrm{Bn}$
2a; $R^{1}=M e, E=C N$
5a; $R=B n, R^{1}=M e, E=C N$
1b; $\mathrm{R}=\mathrm{Hex}$
2b; $R^{1}=M e, E=C O_{2} E t 5 b ; R=H e x, R^{1}=M e, E=C N$
1c; $\mathrm{R}=\mathrm{Bu}$

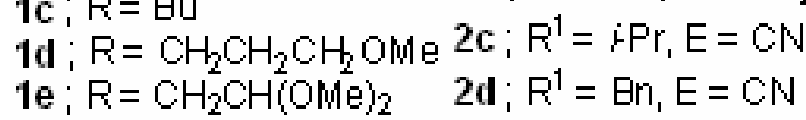
$5 c ; R=B u, R^{1}=M e, E=C N$
5d; $\mathrm{R}=\mathrm{CH}_{2} \mathrm{CH}_{2} \mathrm{CH}_{2}$ OMe, $\mathrm{R}^{1}=\mathrm{Me}, \mathrm{E}=\mathrm{CN}$
if ; $\mathrm{R}=\mathrm{CH}_{2}-p-\mathrm{C}_{6} \mathrm{H}_{4} \mathrm{Cl}$
$5 \mathrm{e} ; \mathrm{R}=\mathrm{CH}_{2} \mathrm{CH}(\mathrm{OMe})_{2}, \mathrm{R}^{1}=\mathrm{Me}, \mathrm{E}=\mathrm{CN}$
5f; $\mathrm{R}=\mathrm{CH}_{2}-p-\mathrm{C}_{6} \mathrm{H}_{4} \mathrm{Cl}, \mathrm{R}^{1}=$ Me, $\mathrm{E}=\mathrm{CN}$
$5 \mathbf{g} ; \mathrm{R}=\mathrm{Bn}, \mathrm{R}^{1}=\mathrm{Me}, \mathrm{E}=\mathrm{CO}_{2} \mathrm{Et}$
5h; $\mathrm{R}=\mathrm{Bn}, \mathrm{R}^{1}=j \mathrm{Pr}, \mathrm{E}=\mathrm{CN}$
5i $\mathrm{R}=\mathrm{Bn}, \mathrm{R}^{1}=\mathrm{Bn}, \mathrm{E}=\mathrm{CN}$

\section{Results and Discussion}

The results are summarized in Table 1 . In the presence of catalytic amounts of $\mathrm{Pd}\left(\mathrm{PPh}_{3}\right)_{4}(5 \mathrm{~mol}$ $\%)$ and triphenylphosphine oxide (10 mol \%), the reaction of 1-benzyl-2-methyleneaziridine 1a $(0.75 \mathrm{mmol})$ with methylmalononitrile $\mathbf{2 a}(0.5 \mathrm{mmol})$ in $\mathrm{THF}$ at $120{ }^{\circ} \mathrm{C}$ for $4 \mathrm{~h}$ gave $5 \mathrm{a}$ in $87 \%$ yield (entry 1). The catalytic system $\mathrm{Pd}(\mathrm{dba})_{2} / \mathrm{PPh}_{3}$ was less effective, and $\mathrm{Pd}_{2}\left(\mathrm{dba}_{3} \cdot \mathrm{CHCl}_{3}\right.$ or $\mathrm{Pd}\left(\mathrm{PPh}_{3}\right)_{2} \mathrm{Cl}_{2}$ did not promote the reaction. The reaction using $\mathrm{Pd}(\mathrm{OAc})_{2} / \mathrm{PPh}_{3}$ as a catalyst gave 5a in a moderate yield. The combination of $\mathrm{Pd}\left(\mathrm{PPh}_{3}\right)_{4}$ and monodentate phosphine ligands such as $\mathrm{PPh}_{3}, \mathrm{P}(\mathrm{O}) \mathrm{Bu}_{3}$, and $\mathrm{P}(\text { o-tolyl })_{3}$, gave 5a in moderate to good yields. In the presence of only $\mathrm{Pd}\left(\mathrm{PPh}_{3}\right)_{4}$, without additional phosphine ligands, 5a was obtained in good yield (80\%). However, even in the presence of $\mathrm{Pd}\left(\mathrm{PPh}_{3}\right)_{4}$, if bidentate ligands such as bis-(diphenylphosphino)methane (dppm), 1,2-bis-(diphenylphosphino)ethane (dppe), 1,3-bis-(diphenylphosphino)propane (dppp) were used as a ligand, only small amounts of $\mathbf{5 a}$ were obtained. The best results were obtained with the catalytic system, $\mathrm{Pd}\left(\mathrm{PPh}_{3}\right)_{4}$ and $\mathrm{P}(\mathrm{O}) \mathrm{Ph}_{3}$. The reactions of 1-hexyl-2-methyleneaziridine $\mathbf{1 b}$ with 2a, and 1-butyl-2-methyleneaziridine $\mathbf{1 c}$ with $\mathbf{2 a}$ afforded $5 \mathbf{b}$ and $\mathbf{5} \mathbf{c}$ in yields of 71 and 
$63 \%$, respectively (entries 2,3 ). The reactions of $\mathbf{1 d}$ with $\mathbf{2 a}$, and $1 \mathbf{e}$ with $\mathbf{2 a}$ proceeded smoothly and the corresponding hydrocarbonation products 5d and 5e were produced in 65 and 79\% yield, respectively (entries 4,5 ). The reaction of 1-p-chlorobenzyl-2-methyleneaziridine, 1f, which has an electron withdrawing group on the nitrogen atom, with $2 \mathbf{a}$ required longer reaction times and gave $\mathbf{5 f}$ in a lower yield (entry 6). The reaction of $\mathbf{1 a}$ with 2-cyanopropionate $\mathbf{2 b}$ afforded $\mathbf{5 g}$ in 63\% yield (entry 7). Other activated methines such as $i$-propylmalononitrile 2c and benzylmalononitrile 2d, upon treatment with 1a, gave products $5 \mathbf{h}$ and $\mathbf{5 i}$ in 71 and $61 \%$ yield, respectively (entries 8,9 ).

Table 1. Palladium catalyzed hydrocarbonation of 1 with $\mathbf{2}^{\mathrm{a}}$

\begin{tabular}{cccccc}
\hline Entry & $\mathbf{1}$ & $\mathbf{2}$ & Time $(\mathrm{h})$ & $\mathbf{3}$ & Yield(\%) $^{\mathrm{b}}$ \\
\hline 1 & $\mathbf{1 a}$ & $\mathbf{2 a}$ & 4 & $\mathbf{5 a}$ & 87 \\
2 & $\mathbf{1 b}$ & $\mathbf{2 a}$ & 5 & $\mathbf{5 b}$ & 71 \\
3 & $\mathbf{1 c}$ & $\mathbf{2 a}$ & 5 & $\mathbf{5 c}$ & 63 \\
4 & $\mathbf{1 d}$ & $\mathbf{2 a}$ & 4 & $\mathbf{5 d}$ & 65 \\
5 & $\mathbf{1 e}$ & $\mathbf{2 a}$ & 4 & $\mathbf{5 e}$ & 79 \\
6 & $\mathbf{1 f}$ & $\mathbf{2 a}$ & 10 & $\mathbf{5 f}$ & 51 \\
7 & $\mathbf{1 a}$ & $\mathbf{2 b}$ & 15 & $\mathbf{5 g}$ & $63(1: 1)^{\mathrm{c}}$ \\
8 & $\mathbf{1 a}$ & $\mathbf{2 c}$ & 5 & $\mathbf{5 h}$ & 71 \\
9 & $\mathbf{1 a}$ & $\mathbf{2 d}$ & 5 & $\mathbf{5 i}$ & 61 \\
\hline
\end{tabular}

${ }^{\mathrm{a}}$ The reaction of $\mathbf{1}(0.75 \mathrm{mmol})$ with $2(0.5 \mathrm{mmol})$ was carried out in the presence of $5 \mathrm{~mol} \%$ of $\mathrm{Pd}\left(\mathrm{PPh}_{3}\right)$ and $10 \mathrm{~mol} \%$ of triphenyl phosphine oxide in THF at $120^{\circ} \mathrm{C}$. bisolated yield based on 2 . ${ }^{\mathrm{c}}$ the diastereomeric ratio of $\mathbf{5 g}$.

Significantly high de's (82\%) were obtained in the reaction of (S)- $N$-(1-naphthylethyl)-2methylene-aziridine $\mathbf{1 h}$ with 2a, although (S)- $N$-(1-phenylethyl)-2-methyleneaziridine $\mathbf{1 g}$ produced only a moderate de (54\%) (eq 4). The absolute stereochemistry of $\mathbf{5 k}$ was determined unambiguously by X-ray analysis and NOE experiments.

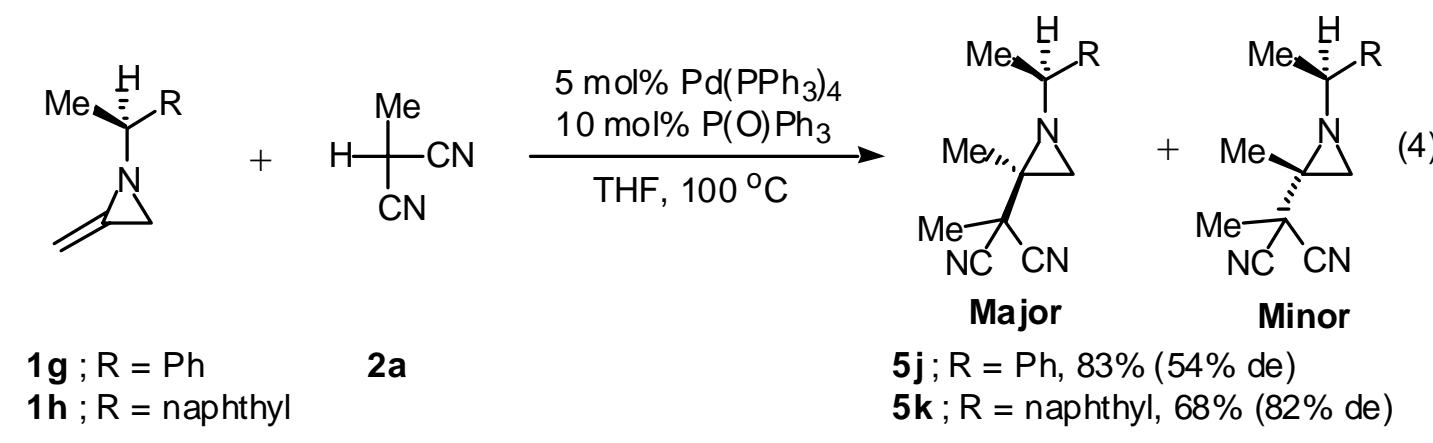

Next, we examined the asymmetric hydrocarbonation of methyleneaziridine 1a with 2a using several chiral phosphine ligands (eq 5). The results are summarized in Table 2. In the presence of 
5 mol.\% of $\mathrm{Pd}(\mathrm{OAc})_{2}$ and 5 mol\% of 1-[1-(acetyloxy)ethyl]-1',2-bis(diphenylphosphino)ferrocene (BPPFOAc), the reaction of 1a with 2a afforded the hydrocarbonation product $\mathbf{5 a}$ in $56 \%$ yield with the enantiomeric excess of $22 \%$ (entry 1). The reaction of 1a with 2a using 1-[1(dimethylamino)ethyl]-2-(diphenylphosphino)ferrocene (BPPFA) instead of BPPFOAc gave 5a with the same level of ee. The reaction of $\mathbf{1 a}$ with $\mathbf{2 a}$ using other ligands, such as 1,1'bis(diphenylphosphino)-2-(1-hydroxyethyl)ferrocene (BPPFOH) and [5-methyl-2-(1methylethyl)cyclohexyl]diphenylphosphine (NMDPP), gave the product 5a in $~ 0 \%$ ee (entries 3 and 4).

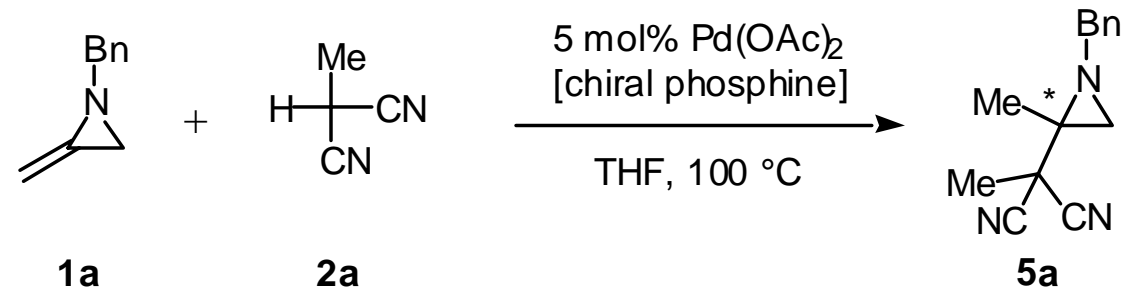

A plausible mechanism for the hydrocarbonation is illustrated in Scheme 1. The oxidative addition of palladium( 0 ) into a $\mathrm{C}-\mathrm{H}$ bond of the pronucleophile $2 \mathbf{a}$ would give the hydridopalladium complex $\mathbf{6} .^{3}$ The hydropalladation of the methyleneaziridines $\mathbf{1}$ with $\mathbf{6}$ would be facilitated by a chelation effect of the nitrogen atom 7, giving the $\mathrm{H}-\mathrm{Pd}$ addition product 8 . Reductive elimination of palladium( 0 ) could then give the hydrocarbonation products $5 .{ }^{4}$

Table 2. Asymmetric hydrocarbonation of $\mathbf{1 a}$ with $\mathbf{2} \mathbf{a}^{\mathrm{a}}$

\begin{tabular}{cccc}
\hline Entry & Ligand & Yield $/ \%^{\mathrm{b}}$ & ee/ ${ }^{\mathrm{c}}$ \\
\hline 1 & BPPFOH & 56 & 22 \\
2 & BPPFA & 55 & 23 \\
3 & BPPFOH & 66 & 0 \\
4 & NMDPP $^{\mathrm{d}}$ & 64 & 0 \\
\hline
\end{tabular}

${ }^{\mathrm{a}}$ The reaction of $\mathbf{1 a}(0.75 \mathrm{mmol})$ with $\mathbf{2 a}(0.5 \mathrm{mmol})$ was carried out in the presence of $5 \mathrm{~mol} \%$ of $\mathrm{Pd}(\mathrm{OAc})_{2}$ and $10 \mathrm{~mol} \%$ of chiral phosphine in THF at $100^{\circ} \mathrm{C}$. ${ }^{\mathrm{b}} \mathrm{NMR}$ yield based on 2 using pxylene as an internal standard. 'Determined by a chiral HPLC analysis ( column: Daicel, chiralcel OD-R ). ${ }^{\mathrm{d}}$ Ten mol\% of NMDPP was used. 

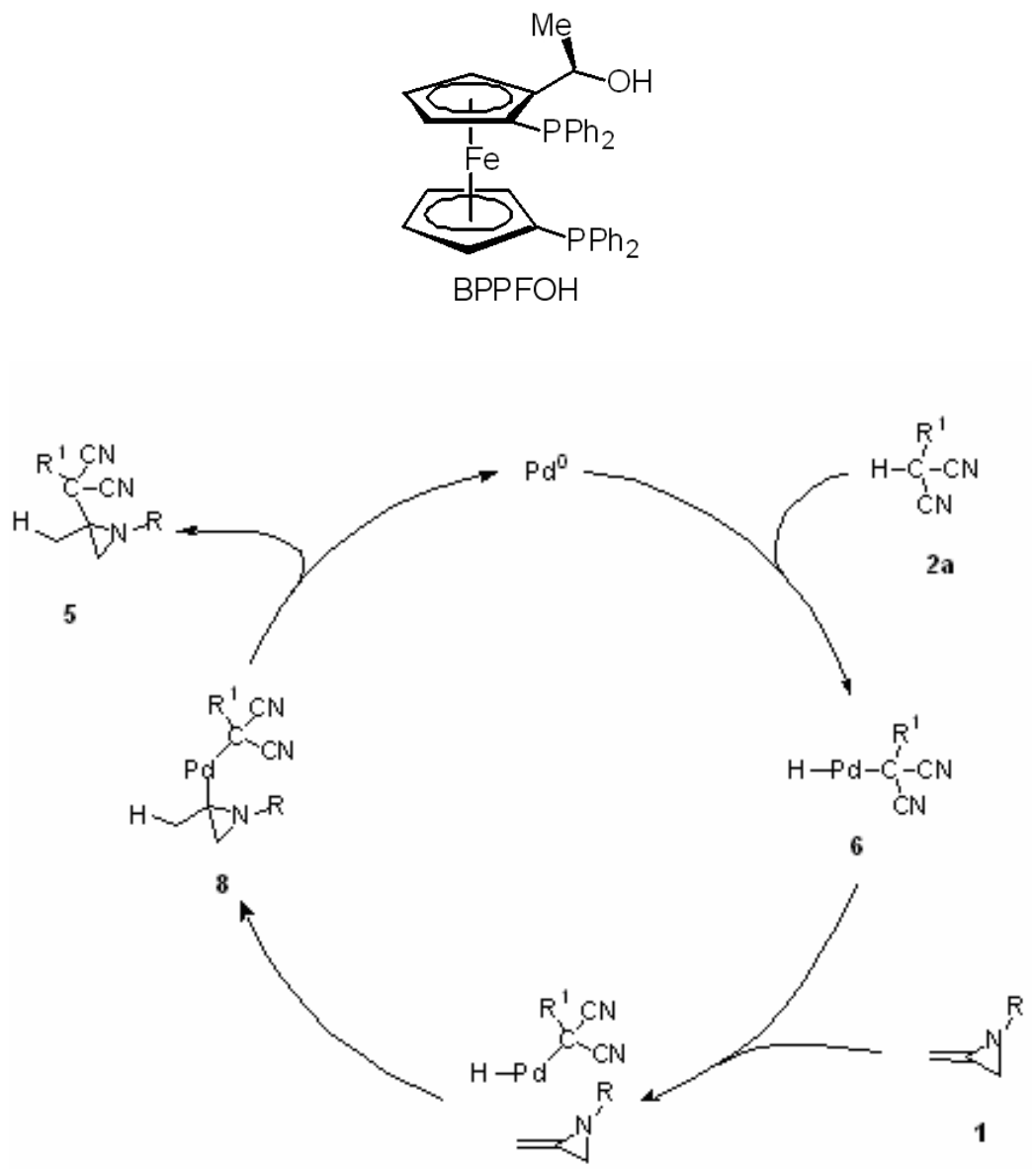

7

\section{Scheme 1}

The reaction with deuterated methylmalononitrile (2a- $d$, 90\% D) substantiated the proposed mechanism. The reaction of $\mathbf{1 a}$ with $\mathbf{2 a}-d$ under the same reaction conditions as above gave 5a- $d$ in $82 \%$ yield, in which the deuterium content was $76 \%$ (eq 6).

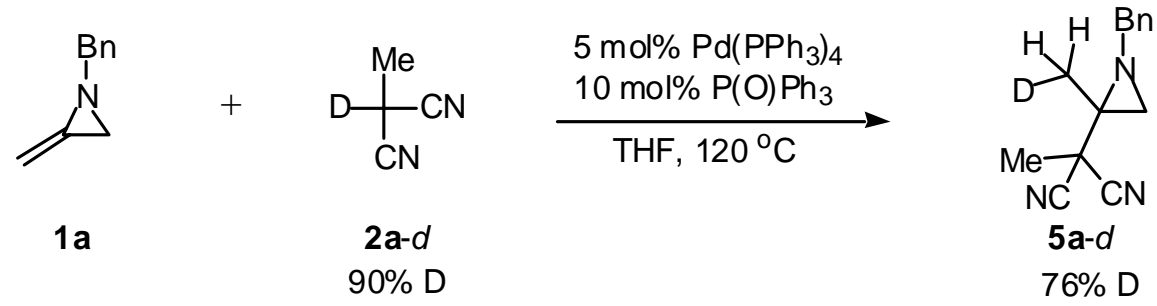

Interestingly, the thermal reaction of $\mathbf{1 a}$ with $\mathbf{2 a}$ without any palladium catalyst in THF at $120{ }^{\circ} \mathrm{C}$ for 4 days gave the vinylic amine 9 in $73 \%$ yield (eq 7). These ring opening reactions of the methyleneaziridine most probably occurred by the nucleophilic addition of the carbanion derived from 2a to the C-3 position of the protonated methyleneaziridine, $\mathbf{1 0}{ }^{1}$ It is now clear that 
the palladium catalyzed and thermal reactions of 2a with 1a take totally different reaction courses; the Lewis acidic Pd(II)-nitrogen interaction (7) leads to 5a while the Brønsted acid $\mathrm{H}^{+}$nitrogen interaction (10) gives $\mathbf{9}$. The addition of carbon pronucleophiles to activated alkenes catalyzed by transition metals, that is the Michael addition, is known. ${ }^{5}$ Recently, we and other groups reported the palladium catalyzed addition of carbon pronucleophiles 2 to unactivated olefins such as allenes, ${ }^{6}$ enynes, ${ }^{7}$ methylenecyclopropanes, ${ }^{8}$ and 1,3 -dienes. ${ }^{9}$ The driving force for these reactions originates in the formation of stable $\pi$-allylpalladium complexes. The present hydrocarbonation reaction does not proceed through the formation of a $\pi$-allylpalladium intermediate, but most probably proceeds via a chelation effect of the nitrogen atom of the aziridine moiety.

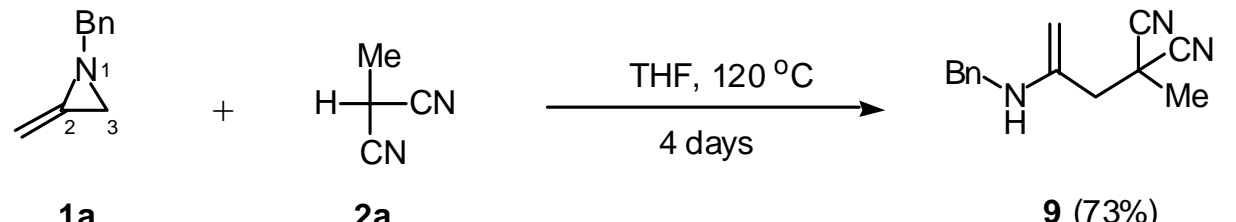

$1 \mathrm{a}$

$2 a$

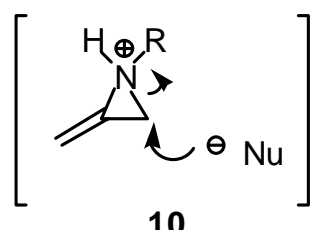

10

\section{Conclusions}

We have developed the direct hydrocarbonation of methyleneaziridines ${ }^{9}$ using carbon pronucleophiles in the presence of a palladium catalyst. The palladium-catalyzed reaction provides geminally disubstituted functionalized aziridines, while traditional reactions give ringopening products upon treatment with nucleophiles.

\section{Experimental Section}

General Procedures. Spectroscopic measurements were carried out with the following instruments: JEOL JNM LA-300 and JEOL $\alpha-500\left({ }^{1} \mathrm{H}-\right.$ and ${ }^{13} \mathrm{C}$ NMR), SHIMADZU FTIR8200A (FT-IR), HITACHI M-2500s (HRMS). All methyleneaziridines were prepared following the reported procedure. ${ }^{10}$ Daicel Chiralcel OD-R was used to analyze the enantiomeric excess of 5a. 
General procedure for the addition of the active methyne 1 to methyleneaziridines 4

To a solution of $\mathrm{Pd}\left(\mathrm{PPh}_{3}\right)_{4}$ (28.9 mg, $\left.0.025 \mathrm{mmol}\right)$, triphenylphosphine oxide (13.9 $\mathrm{mg}, 0.05$ mmol) and active methyne $1(0.5 \mathrm{mmol})$ in THF $(1 \mathrm{~mL})$ was added methyleneaziridine 4 (0.75 mmol) under Ar atmosphere in pressure vial. After heating at $120^{\circ} \mathrm{C}$ for $4-15$ hours, the reaction mixture was filtered through a short Florisil column using ethyl acetate as an eluent. Separation by passing through a Florisil column using $n$-hexane-ethyl acetate as eluent.

2-(1-Benzyl-2-methylaziridin-2-yl)-2-methylmalononitrile (5a). Pale yellow oil: IR (neat) 3062, 3031, 2997, 2935, 2858, 2250, 1496, 1454, 1392, 1342, 1245, 1182, 1159, 1126, 1076, 1028, 736, $698 \mathrm{~cm}^{-1}$; ${ }^{1} \mathrm{H}$ NMR (500 MHz, $\mathrm{CDCl}_{3}$ ) $\delta 1.48$ (s, 1H), 1.53 (s, 3H), 1.64 (s, 3H), 2.31 (s, $1 \mathrm{H}), 3.70$ (d, $J=2.5 \mathrm{~Hz}, 2 \mathrm{H}), 7.25-7.39$ (m, 5H); ${ }^{13} \mathrm{C}$ NMR (125 MHz, $\mathrm{CDCl}_{3}$ ) $\delta 11.64,20.79$, 37.14, 39.64, 41.87, 56.49, 115.03, 115.22, 127.34, 127.91, 128.44, 138.50. HRMS (EI) Calcd for $\mathrm{C}_{14} \mathrm{H}_{15} \mathrm{~N}_{3} ; \mathrm{m} / \mathrm{z}$ 225.1266: found, 225.1271.

2-(1-Hexyl-2-methylaziridin-2-yl)-2-methylmalononitrile (5b). Pale yellow oil: IR (neat) 2931, 2858, 2250, 1456, 1392, 1377, 1342, 1182, 1164, $1126 \mathrm{~cm}^{-1} ;{ }^{1} \mathrm{H}$ NMR (300 MHz, $\mathrm{CDCl}_{3}$ ) $\delta 0.89(\mathrm{t}, J=6.9 \mathrm{~Hz}, 3 \mathrm{H}), 1.29-1.57(\mathrm{~m}, 12 \mathrm{H}), 1.72(\mathrm{~s}, 3 \mathrm{H}), 2.19(\mathrm{~s}, 1 \mathrm{H}), 2.47$ (td, $J=6.6$ and 2.0 $\mathrm{Hz}, 2 \mathrm{H}) ;{ }^{13} \mathrm{C} \mathrm{NMR}\left(75 \mathrm{MHz}, \mathrm{CDCl}_{3}\right.$ ) 11.22, 13.98, 20.64, 22.54, 26.83, 30.25, 31.61, 36.97, 39.68, 41.37, 52.91, 115.30, 115.34. HRMS (EI) Calcd for $\mathrm{C}_{13} \mathrm{H}_{21} \mathrm{~N}_{3} ; \mathrm{m} / \mathrm{z}$ 219.1735: found, 219.1727.

2-(1-Butyl-2-methylaziridin-2-yl)-2-methylmalononitrile (5c). Pale yellow oil: IR (neat) 2958, 2935, 2864, 2252, 1456, 1392, 1377, 1340, 1244, 1184, 1168, 1145, 1126, 1070, $1045 \mathrm{~cm}^{-1} ;{ }^{1} \mathrm{H}$ NMR (300 MHz, $\left.\mathrm{CDCl}_{3}\right) \delta 0.93(\mathrm{t}, J=6.9 \mathrm{~Hz}, 3 \mathrm{H}), 1.29(\mathrm{~s}, 1 \mathrm{H}), 1.32-1.62(\mathrm{~m}, 7 \mathrm{H}), 1.72(\mathrm{~s}$, 3H), 2.19 (s, 1H), 2.48 (t, $\left.J=6.6 \mathrm{~Hz}, 2 \mathrm{H}) ;{ }^{13} \mathrm{C} \mathrm{NMR} \mathrm{(75} \mathrm{MHz,} \mathrm{CDCl}_{3}\right) \delta 11.13,13.86,20.27$, 20.60, 32.33, 36.92, 39.68, 41.31, 52. 52, 115.26, 115.30. HRMS (EI) Calcd for $\mathrm{C}_{11} \mathrm{H}_{17} \mathrm{~N}_{3} ; \mathrm{m} / \mathrm{z}$ 191.1422: found, 191.1422.

2-[1-(3-Methoxypropyl)-2-methylaziridin-2-yl]-2-methylmalononitrile (5d). Pale yellow oil: IR (neat) 2931, 2873, 2831, 2249, 1452, 1392, 1342, 1245, 1224, 1186, 1164, $1043 \mathrm{~cm}^{-1} ;{ }^{1} \mathrm{H}$ NMR (300 MHz, CDCl $) \delta 1.33(\mathrm{~s}, 1 \mathrm{H}), 1.50$ (s, 3H), 1.73 (s, 3H), 1.78-1.89 (m, 2H), 2.22 (s, 1H), 2.47-2.66 (m, 2H), 3.34 (s, 3H), 3.51 (t, $J=6.6 \mathrm{~Hz}, 2 \mathrm{H}) ;{ }^{13} \mathrm{C}$ NMR $\left(75 \mathrm{MHz}, \mathrm{CDCl}_{3}\right) \delta$ 11.23, 20.68, 30.34, 37.13, 39.74, 41.41, 49.55, 58.59, 70.11, 115.27, 115.29. HRMS (EI) Calcd for $\mathrm{C}_{11} \mathrm{H}_{17} \mathrm{~N}_{3} \mathrm{O}$, m/z 207.1372: found, 207.1376.

2-[1-(2,2-Dimethoxyethyl)-2-methylaziridin-2-yl]-2-methylmalononitrile (5e). Pale yellow oil: IR (neat) 2993, 2945, 2912, 2835, 1454, 1888, 1346, 1313, 1247, 1188, 1166, 1134, 1076, 968, $854 \mathrm{~cm}^{-1}$; ${ }^{1} \mathrm{H}$ NMR (300 MHz, $\mathrm{CDCl}_{3}$ ) $\delta 1.41$ (s, 1H), 1.48 (s, 3H), $1.74(\mathrm{~s}, 3 \mathrm{H}), 2.25$ (s, $1 \mathrm{H}), 2.57-2.72(\mathrm{~m}, 2 \mathrm{H}), 3.43(\mathrm{~d}, J=2.4 \mathrm{~Hz}, 6 \mathrm{H}), 4.54(\mathrm{t}, J=5.4 \mathrm{~Hz}, 1 \mathrm{H}) ;{ }^{13} \mathrm{C}$ NMR $(75 \mathrm{MHz}$, $\left.\mathrm{CDCl}_{3}\right) \delta 11.86,20.64,36.81,39.59,41.21,54.14,54.54,54.73,104.44,115.06,115.16$. HRMS (EI) Calcd for $\mathrm{C}_{11} \mathrm{H}_{17} \mathrm{~N}_{3} \mathrm{O}_{2}, \mathrm{~m} / \mathrm{z}$ 223.1321: found, 223.1327.

2-[1-(4-Chloro-benzyl)-2-methylaziridin-2-yl]-2-methylmalononitrile (5f). Pale yellow oil: IR (neat) 2977, 2937, 2860, 2250, 1596, 1492, 1454, 1409, 1340, 1245, 1182, 1161, 1087, 1014, 
842, 806, $773 \mathrm{~cm}^{-1} ;{ }^{1} \mathrm{H}$ NMR (300 MHz, $\mathrm{CDCl}_{3}$ ) $\delta 1.48$ (s, 1H), 1.54 (s, 3H), 1.67 (s, 3H), 2.32 (s, 1H), 3.67 (q, $J=13.7 \mathrm{~Hz}, 2 \mathrm{H}), 7.32$ (s, 4H); ${ }^{13} \mathrm{C}$ NMR $\left(75 \mathrm{MHz}, \mathrm{CDCl}_{3}\right) \delta 11.60,20.83$, 37.12, 39.65, 41.92, 55.75, 114.93, 115.08, 128.58, 129.17, 133.04, 136.98. HRMS (EI) Calcd for $\mathrm{C}_{14} \mathrm{H}_{14} \mathrm{~N}_{3} \mathrm{Cl}, \mathrm{m} / \mathrm{z}$ 259.0876: found, 259.0873.

(1-Benzyl-2-methylaziridin-2-yl)-cyanomethylacetic acid ethyl ester (5g). Diastereoisomer A. Pale yellow oil: IR (neat) 2985, 2240, 1741, 1452, 1257, 1174, 1153, 1114, 1064, 1016, 736, 698 $\mathrm{cm}^{-1} ;{ }^{1} \mathrm{H}$ NMR (300 MHz, $\left.\mathrm{CDCl}_{3}\right) \delta 1.29$ (t, $\left.J=7.1 \mathrm{~Hz}, 3 \mathrm{H}\right), 1.34$ (s, 1H), 1.39 (s, 3H), 1.49 (s, 3H), 2.30 (s, 1H), 3.53-3.82 (m, 2H), 4.18-4.27 (m, 2H), 7.23-7.41 (m, 5H); ${ }^{13} \mathrm{C}$ NMR (75 $\left.\mathrm{MHz}_{\mathrm{CDCl}}\right) \delta 12.34,13.96,19.10,37.28,41.94,50.69$, 56.41, 62.56, 119.01, 126.99, 127.86, 128.27, 139.36, 167.55; HRMS (EI) Calcd for $\mathrm{C}_{16} \mathrm{H}_{20} \mathrm{~N}_{2} \mathrm{O}_{2}: \mathrm{m} / \mathrm{z}$ 272.1525: found, 272.1528.

Diastereoisomer B. Pale yellow oil: IR (neat) 2932, 2241, 1741, 1452, 1259, 1153, 1114, 1066, 1018, 734, $698 \mathrm{~cm}^{-1}$; ${ }^{1} \mathrm{H}$ NMR (300 MHz, $\mathrm{CDCl}_{3}$ ) $\delta 1.29$ (t, $\left.J=7.1 \mathrm{~Hz}, 3 \mathrm{H}\right), 1.34$ (s, $\left.1 \mathrm{H}\right), 1.43$ (s, 3H), 1.51 (s, 3H), 2.25 (s, 1H), 3.56 (d, $J=13.9 \mathrm{~Hz}, 1 \mathrm{H}), 3.80$ (d, $J=13.9 \mathrm{~Hz}, 1 \mathrm{H}), 4.21$ (q, $J$ $=7.1 \mathrm{~Hz}, 2 \mathrm{H}), 7.24-7.41(\mathrm{~m}, 5 \mathrm{H}) ;{ }^{13} \mathrm{C} \mathrm{NMR}\left(75 \mathrm{MHz}, \mathrm{CDCl}_{3}\right) \delta 12.94,13.90,19.03,36.87$, 41.78, 50.42, 56.45, 62.62, 119.16, 126.99, 127.88, 128.27, 139.33, 168.20; HRMS (EI) Calcd for $\mathrm{C}_{16} \mathrm{H}_{20} \mathrm{~N}_{2} \mathrm{O}_{2}: m / z$ 272.1525: found, 272.1529 .

2-(1-Benzyl-2-methylaziridin-2-yl)-2-isopropyl-malononitrile (5h). Pale yellow oil: IR (neat) 3087-2858, 2249, 1497, 1454, 1394, 1340, 1242, 1147, 734, $698 \mathrm{~cm}^{-1}$; ${ }^{1} \mathrm{H}$ NMR (300 MHz, $\left.\mathrm{CDCl}_{3}\right) \delta 1.17-1.23(\mathrm{~m}, 6 \mathrm{H}), 1.45$ (s, $\left.1 \mathrm{H}\right), 1.48$ (s, 3H), 2.29-2.38 (m, 2H), 3.45 (d, $J=13.8 \mathrm{~Hz}$, 1H), 3.95 (d, $J=14.1 \mathrm{~Hz}, 1 \mathrm{H}), 7.24-7.42$ (m, 5H); ${ }^{13} \mathrm{C}$ NMR (75 MHz, $\mathrm{CDCl}_{3}$ ) $\delta 11.12,17.95$, 18.77, 33.44, 37.81, 40.32, 53.01, 56.01, 113.45, 113.99, 127.17, 127.85, 128.31, 138.30. HRMS (EI) Calcd for $\mathrm{C}_{16} \mathrm{H}_{19} \mathrm{~N}_{3}, \mathrm{~m} / \mathrm{z}$ 253.1579: found, 253.1581.

2-Benzyl-2-(1-benzyl-2-methyl-aziridin-2-yl)-malononitrile (5i). White solid: IR (KBr) 30852889, 2253, 1604, 1496, 1456, 1398, 1359, 1336, 1249, 1228, 1151, 1028, 740, $704 \mathrm{~cm}^{-1} ;{ }^{1} \mathrm{H}$ NMR (300 MHz, $\left.\mathrm{CDCl}_{3}\right) \delta 1.50$ (s, 1H), 1.62 (s, 3H), 2.26 (s, 1H), 2.92 (d, $\left.J=13.5 \mathrm{~Hz}, 1 \mathrm{H}\right)$, 3.12 (d, $J=13.5 \mathrm{~Hz}, 1 \mathrm{H}), 3.73$ (s, 2H), 7.23-7.43 (m, 10H); ${ }^{13} \mathrm{C}$ NMR (75 MHz, $\left.\mathrm{CDCl}_{3}\right) \delta 12.20$, 37.73, 39.36, 41.94, 48.11, 56.45, 113.83, 114.15, 127.41, 128.00, 128.45, 128.50, 128.77, 130.08, 132.41, 138.51. HRMS (EI) Calcd for $\mathrm{C}_{20} \mathrm{H}_{19} \mathrm{~N}_{3}, \mathrm{~m} / \mathrm{z}$ 301.1579: found, 301.1583.

2-Methyl-2-[2-methyl-1-(1-Phenyl-ethyl)-aziridine-2-yl]-malononitrile(5j).

Major diastereoisomer. Pale yellow oil: IR (neat) 2974, 2250, 1492, 1450, 1394, 1373, 1340, 1168, 1128, 1110, 1089, 1028, 1158, 702; ${ }^{1} \mathrm{H}$ NMR (500 MHz, $\left.\mathrm{CDCl}_{3}\right) \delta 1.28$ (s, 1H), 1.41 (d, $J$ $=6.6 \mathrm{~Hz}, 3 \mathrm{H}), 1.63(\mathrm{~s}, 3 \mathrm{H}), 1.79(\mathrm{~s}, 3 \mathrm{H}), 2.10(\mathrm{~s}, 1 \mathrm{H}), 3.19(\mathrm{q}, J=6.5 \mathrm{~Hz}, 1 \mathrm{H}), 7.24-7.41(\mathrm{~m}$, $5 \mathrm{H}) ;{ }^{13} \mathrm{C}$ NMR $\left(125 \mathrm{MHz}, \mathrm{CDCl}_{3}\right) \delta 11.38,20.74,25.13,35.80,39.96,42.95,61.42,115.30$, 115.31, 126.75, 127.28, 128.43, 144.04; HRMS (EI) Calcd for $\mathrm{C}_{15} \mathrm{H}_{17} \mathrm{~N}_{3}$ : m/z 239.1422; found 239.1420 .

Minor diastereoisomer. Pale yellow oil: IR (neat) 2974, 2250, 1492, 1452, 1392, 1377, 1340, 1163, 1128, 1099, 1068, 1028, 758, 702; ${ }^{1} \mathrm{H}$ NMR (300 MHz, $\left.\mathrm{CDCl}_{3}\right) \delta 1.34$ (s, 3H), 1.41 (d, $J=$ $6.4 \mathrm{~Hz}, 3 \mathrm{H}), 1.46$ (s, 1H), 1.48 (s, 3H), 2.29 (s, 1H), 3.18 (q, J = 6.4 Hz, 1H), 7.21-7.38 (m, 5H); 
${ }^{13} \mathrm{C}$ NMR $\left(125 \mathrm{MHz}, \mathrm{CDCl}_{3}\right) \delta 11.67,21.09,24.07,35.89,39.91,42.40,62.30,114.89,115.30$, 126.80, 127.54, 128.57, 144.47; HRMS (EI) Calcd for $\mathrm{C}_{15} \mathrm{H}_{17} \mathrm{~N}_{3}: \mathrm{m} / \mathrm{z}$ 239.1422; found 239.1419.

The stereochemistry of $\mathbf{5} \mathbf{j}$ was determined by NOE experiment as shown in Figure 1.

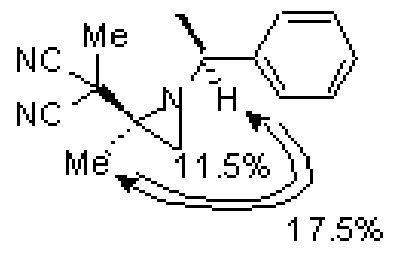

(a) major

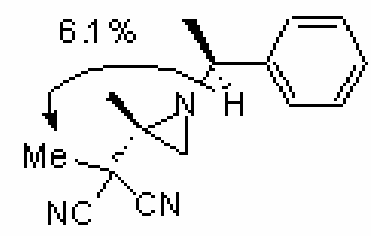

(b) minor

Figure 1. NOE experimet of $\mathbf{5 j}$.

\section{2-Methyl-2-[2-methyl-1-(1-naphthalen-1-yl-ethyl)-aziridine-2-yl]-malononitrile (5k)}

Major diastereoisomer. White solid: IR (KBr) 2977, 2931, 2247, 1596, 1473, 1452, 1340, 1230, 118, 1110, 779; ${ }^{1} \mathrm{H}$ NMR (300 MHz, $\left.\mathrm{CDCl}_{3}\right) \delta 1.29$ (s, $\left.1 \mathrm{H}\right), 1.59$ (d, $J=6.4 \mathrm{~Hz}, 3 \mathrm{H}$ ), 1.75 (s, 3H), 1.87 (s, 3H), 2.22 (s, 1H), 3.97 (s, 1H), 7.46-7.54 (m, 4H), 7.78(d, $J=8.2 \mathrm{~Hz}, 1 \mathrm{H}), 7.87-$ 8.08 (m, 2H); ${ }^{13} \mathrm{C}$ NMR (125 MHz, $\left.\mathrm{CDCl}_{3}\right)$ 11.37, 20.97, 24.47, 36.16, 40.29, 43.59, 57.54, 115.37, 115.38, 122.82, 124.32, 125.40, 125.89, 125.93, 127.69, 129.12, 130.58, 133.87, 139.66; HRMS (EI) Calcd for $\mathrm{C}_{19} \mathrm{H}_{19} \mathrm{~N}_{3}: \mathrm{m} / \mathrm{z}$ 289.1579; found 289.1580.

Minor diastereoisomer. Pale yellow oil: IR (neat) 2972, 2952, 2247, 1596, 1450, 1394, 1340, 1247, 1178, 1155, 802, 779; ${ }^{1} \mathrm{H}$ NMR (300 MHz, $\mathrm{CDCl}_{3}$ ) $\delta 1.26$ (s, 3H), 1.56-1.60 (m, 7H), 2.43 (s, $1 \mathrm{H}), 3.92$ (s, 1H), 7.46-7.54 (m, 4H), 7.77-7.90 (m, 3H); ${ }^{13} \mathrm{C} \mathrm{NMR}\left(75 \mathrm{MHz}, \mathrm{CDCl}_{3}\right)$ 11.71, 21.34, 23.43, 36.35, 40.17, 42.80, 57.69, 115.22, 115.30, 122.91, 124.86, 125.42, 125.57, 125.92, 127.84, 129.07, 129.97, 133.92, 140.08; HRMS (EI) Calcd for $\mathrm{C}_{19} \mathrm{H}_{19} \mathrm{~N}_{3}$ : m/z 289.1579; found 289.1582.

The stereochemistry of $\mathbf{5 k}$ was determined by NOE experiment as shown in Figure 2.

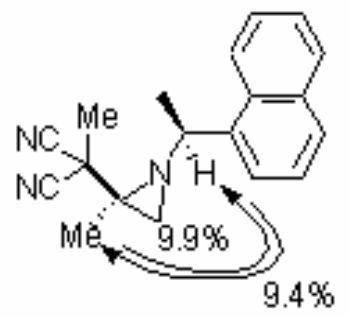

major diaster eoisomer

Figure 2. NOE experiment of 5k. 
We could not measure an NOE experiment for the minor diastereoisomer of $5 \mathbf{k}$ because of overlap of the peaks. The ORTEP drawing of the major diastereomer of $\mathbf{5 k}$ is shown in Figure 3.

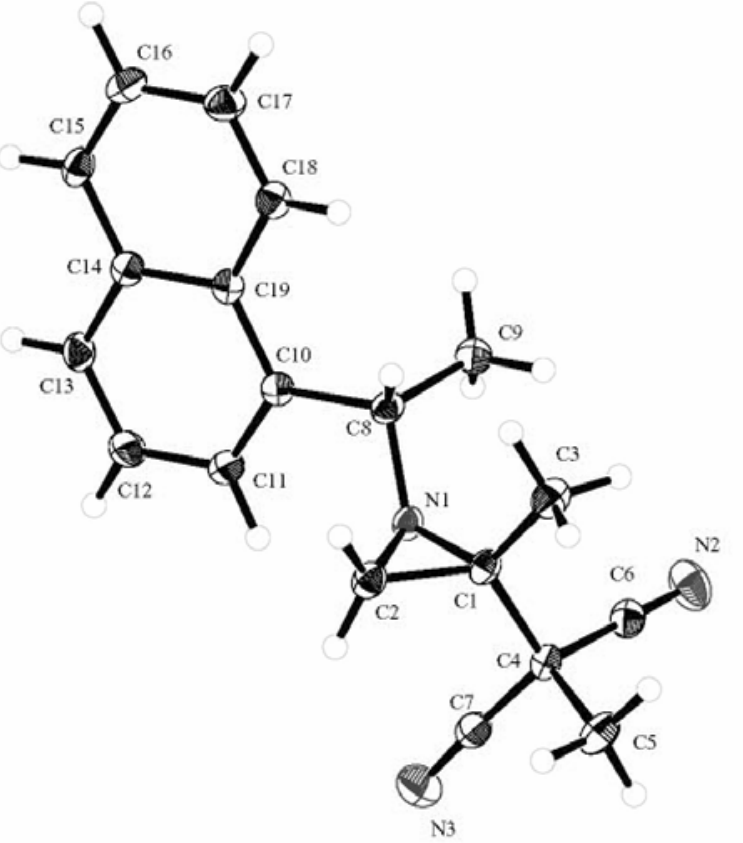

Figure 3. ORTEP drawing of the major isomer of $\mathbf{5 k}$.

2-[1-(Benzylamino-methyl)-vinyl]-2-methyl-malononitrile (9). Pale yellow oil: IR (neat) 3307, 2931, 2247, 1651, 1496, 1454, 1404, 1344, 1201, 1114, 981, 808, 727, 696; ${ }^{1} \mathrm{H}$ NMR (500 $\left.\mathrm{MHz}, \mathrm{CDCl}_{3}\right) \delta 1.67(\mathrm{~s}, 1 \mathrm{H}), 2.71(\mathrm{td}, J=1.5$ and $15.5 \mathrm{~Hz}, 1 \mathrm{H}), 3.21(\mathrm{td}, J=1.5$ and $15.5 \mathrm{~Hz}$, $1 \mathrm{H}$ ), 4.14 (ddd, $J=2.0,3.5$ and $39.0 \mathrm{~Hz}, 2 \mathrm{H}), 4.76$ (d, $J=4.2 \mathrm{~Hz}, 2 \mathrm{H}), 7.21-7.33(\mathrm{~m}, 5 \mathrm{H}) ;{ }^{13} \mathrm{C}$ NMR (125 MHz, $\mathrm{CDCl}_{3}$ ) 824.64, 39.16, 39.46, 44.89, 84.59, 120.57, 126.16, 126.75, 127.42, 128.67, 128.82, 142.64; HRMS (EI) Calcd for $\mathrm{C}_{14} \mathrm{H}_{15} \mathrm{~N}_{3}: \mathrm{m} / \mathrm{z} 225.1266$. found: 225.1261.

\section{References and Notes}

1. For ring opening reactions of methyleneaziridines. (a) Quast, H.; Weise Velez, C. A. Angew. Chem., Int. Ed. Engl. 1974, 13, 342. (b) Hayes, J. F.; Shipman, M.; Twin, H. Chem. Commun. 2000, 1791. (c) Hayes, J. F.; Shipman, M.; Twin, H. Chem. Commun. 2001, 1784. (d) Hayes, J. F.; Shipman, M.; Twin, H. J. Org. Chem. 2002, 67, 935. (e) Ince, J.; Shipman, M.; Ennis, D. S. Tetrahedron Lett. 1997, 38, 5887. (f) Ennis, D. S.; Ince, J.; Rahman, S.; 
Shipman, M. J. Chem. Soc., Perkin Trans. 1 2000, 2047. (g) Bottini, A. T.; Roberts, J. D. J. Am. Chem. Soc. 1957, 79, 1462. (h) Crandall, J. K.; Crawley, L. C.; Komin, J. B. J. Org. Chem. 1975, 40, 2045. (i) Bottini, A. T.; Roberts, J. D. J. Am. Chem. Soc. 1962, 84, 195. (j) Jongejan, E.; Steinberg, H.; De Boer, T. J. Recl. Trav. Chim. Pays-Bas. 1978, 97, 146. (k) Jongejan, E.; Steinberg, H.; De Boer, T. J. Recl. Trav. Chim. Pays-Bas. 1979, 98, 66.

2. Oh, B. H.; Nakamura, I.; Yamamoto, Y. Tetrahedron Lett. 2002, 43, 9625.

3. The anionic structure, $\mathrm{H}-\mathrm{Pd}^{+}{ }^{-} \mathrm{CR}_{3}$, is also conceivable.

4. $\quad \mathrm{Pd}$ catalyzed ring expansion reactions of methyleneaziridine with carbon monoxide $: \operatorname{Pd}(0)$ catalyst inserts into N-C2 bond of methyleneaziridines : Alper, H.; Hamel, N. Tetrahedron Lett. 1987, 28, 3237.

5. (a) Naota, T.; Taki, H.; Mizuno, M.; Murahashi, S.-I. J. Am. Chem. Soc. 1989, 111, 5954.

(b) Paganelli, S.; Schionato, A.; Botteghi, C. Tetrahedron Lett. 1991, 32, 2807. (c) Sawamura, M.; Hamashita, H.; Ito, Y. J. Am. Chem. Soc. 1992, 114, 8295. (d) Murahashi, S.-I.; Naota, T.; Taki, H.; Mizuno, M.; Takaya, H.; Komiya, S.; Mizuho, Y.; Oyasato, N.; Hiraoka, M.; Hirano, M.; Fukuoka, A. J. Am. Chem. Soc. 1995, 117, 12436. (e) GómezBengoa, E.; Cuerva, J. M.; Mateo, C.; Echavarren, A. J. Am. Chem. Soc. 1996, 118, 8553.

6. (a) Yamamoto, Y.; Al-Masum, M.; Asao, N. J. Am. Chem. Soc. 1994, 116, 6019. (b) Trost, B. M.; Gerusz, V. J. J. Am. Chem. Soc. 1995, 117, 5156. (c) Basson, L.; Goré, J.; Cazes, B. Tetrahedron Lett. 1995, 36, 3853. (d) Yamamoto, Y.; Al-Masum, M.; Fujiwara, N.; Asao, N. Tetrahedron Lett. 1995, 36, 2811. (e) Yamamoto, Y.; Al-Masum, M.; Fujiwara, N. J. Chem. Soc., Chem. Commun. 1996, 381. (f) Yamamoto, Y.; Al-Masum, M.; Takeda, A. J. Chem. Soc., Chem. Commun. 1996, 831. (g) Grigg, R.; Kongathip, N.; Kongathip, B.; Luangkamin, S.; Dondas, H. A. Tetrahedron 2001, 57, 9187.

7. Gevorgyan, V.; Kadowaki, C.; Salter, M. M.; Kadota, I.; Saito, S.; Yamamoto, Y. Tetrahedron 1997, 53, 9097.

8. (a) Tsukada, N.; Shibuya, A.; Nakamura, I.; Yamamoto, Y. J. Am. Chem. Soc. 1997, 119, 8123. (b) Tsukada, N.; Shibuya, A.; Nakamura, I.; Kitahara, H.; Yamamoto, Y. Tetrahedron 1999, 55, 8833. (c) Nakamura, I.; Saito, S.; Yamamoto, Y. J. Am. Chem. Soc. 2000, 122, 2661.

9. (a) Takahashi, K.; Miyake, A.; Hata, G. Bull. Chem. Soc. Jpn. 1972, 45, 1183. (b) Baker, R.; Popplestone, R. J. Tetrahedron Lett. 1978, 38, 3575. (c) Andell, O. S.; Bäckvall, J.-E.; Moberg, C. Acta Chem. Scand. Ser. B. 1986, 40, 184. (d) Jolly, P. W.; Kokel, N. Synthesis 1990, 771. (e) Trost, B. M.; Zhi, L. Tetrahedron Lett. 1992, 33, 1831.

10. Preparation of 2-methyleneaziridines: (a) Pollard, C. B.; Parcell, R. F. J. Am. Chem. Soc. 1951, 73, 2925. (b) Bingham, E. M.; Gilbert, C. J. J. Org. Chem. 1975, 40, 224. (c) Atkinson, R. S.; Malpass, J. R. Tetrahedron Lett. 1975, 4305. (d) Ince, J.; Ross, T. M.; Shipman, M.; Slawin, A. M. Z.; Ennis, D. S. Tetrahedron 1996, 52, 7037. (e) Ince, J.; Ross, 
T. M.; Shipman, M.; Ennis, D. S. Tetrahedron: Asymmetry 1996, 7, 3397. (f) De Kimpe, N.; De Smaele, D. Skonyi, Z. J. Org. Chem. 1997, 62, 2448. 